CASE OF

\title{
POPLITEAL ANEURISM
}

\section{TREATED BY COMPRESSION,}

WI'TH SOME REMARKS UPON THIS METHOD OF TREATING ANEURISM, AND A LIST OF THE CASES IN WHICH IT HAS BEEN

EMPLOYED IN DUBLIN.

\author{
BY \\ O'BRYEN BELLINGHAM, M.D. \\ FELLOW OF, AND MEMBER OF THE COURT OF EXAMINERS OF, \\ THE ROYAL COLLEGR OF SURGEONS IN IRELAND, \\ SURGEON TO ST. VINCENT'S HOBPITAL, ETC., ETC. \\ COMMUNICATED BY \\ SIR BENJAMIN BRODIE, BART., F.R.S.
}

Received February 7th.-Read June 10th, 1851.

Charles Maher, æt. 42, a labourer from the county of Carlow, was admitted into St. Vincent's Hospital, under the care of Mr. Bellingham, Nov. 26th, 1850, labouring under popliteal aneurism on the right side. He states that he was formerly in the army, and served eight years in the 19th Regiment of Infantry, three of which were spent in the West Indies. He enjoyed very good health, with the exception of an attack of dysentery, under which he suffered there; he was discharged ten years ago, owing to defective vision, caused by some opacity of the cornea of the right eye. He has been engaged since then in agricultural labour; latterly, his employment has consisted in spade labour, in deepening the bed of a river, in a hard soil, where he was sometimes up to his knees in water. He attributes the disease to this work, the right lower extremity being prin- 
cipally employed in it ; he says he never received a strain, or other injury.

He states that, in June last, he first began to feel pain, which was referred to the centre of the sole of the right foot; that about a month afterwards, the limb swelled from the ankle to the knee, and became nearly double the size of the other, accompanied by cramp in the leg and pain in the ham, which he observed to be fuller than the other. About three weeks ago, he noticed the pulsation for the first time; he continued to work up to a week ago; and has made several applications to the part, among the rest a blister, with the hope of relief. He then applied to a medical man, who recommended him to come up to Dublin to be treated, as he could not afford to lie up in the country.

The popliteal region of the right limb is occupied by a tumour, which has a very strong and heaving impulse, both posteriorly and laterally, elevating the head when the stethoscope is laid on it; on pressing upon the artery in the groin, the pulsation ceases, and the tumour collapses in a certain degree; and, on auscultation over it, a short, harsh, bruit de souffet is heard. The tumour completely fills the popliteal space, is about the size of an orange, measuring three inches from above downwards, and three inches and a half transversely; the ham-string tendons are much stretched, and the integuments covering it are discoloured from the effects of a blister which had been applied in the country. The limb, at this part, measures fifteen inches and $a$ half in circumference, the opposite limb, at the same point, fourteen inches. The limb, from the ham downwards, looks to be somewhat larger than the other; and, on measurement at the calf, is found to be fourteen inches and a half, while on the other side it is only thirteen inches and a half. $\mathrm{He}$ is a stout and muscular man; his general health very good; the action of the heart regular; no increased impulse; pulse 64, strong.

November 30th.-The patient was directed to remain constantly in bed, to take half a drachm of the Pulv. Jalap. 
Comp. every night, and to be restricted to the following diet:

Two ounces of bread and two ounces of milk for breakfast. The same for supper.

Two ounces of bread, two ounces of milk, and two ounces of meat for dinner.

December 3d.- The patient has not exceeded, so far as I can learn, the dietary prescribed; he makes no complaint, except of thirst, and of it only when questioned. The pulse is smaller and softer, and the limb at the site of the aneurism has diminished half an inch in circumference.

4th.-Compression commenced to-day, at 11 o'clock, a.m., by means of two instruments, one upon the artery as it crosses the horizontal ramus of the pubes, the other at the lower third of the thigh; the pulse at this time being soft, small, and compressible, and beating only 56 in the minute. Little congestion of the limb was caused by the pressure; and the sac became, in a great measure, flaccid. The compression was maintained during the day, so as to check pulsation in the tumour; at each visit to the patient, however, a considerable impulse was felt in it, as he could not tell when the pulsation was checked; and in the slight movements in bed, the artery slipped from under the pad.

Half-past eight o'clock, p.m.-The aneurism does not collapse now when the pressure is maintained, but its outlines can be distinctly felt; the patient's skin is cool; he makes no complaint of pain, but says he is very thirsty; and he was allowed a little whey. The patient by this time appeared to understand the object of the compression, and could tell when the pulsation in the tumour was checked; the management of the compressing instruments was, therefore, left to him during the night, and he was directed to alternate the points of pressure whenever pain was experienced, and not to allow any impulse in the aneurism. I had, I should have observed, marked with ink, upon the patient's thigh, the points upon which he was to keep the pad of the compressing instruments, and this part of the limb was directed to be dusted occasionally with flour. 
5th.-The patient remained awake during the night, and says that he continued the pressure so as not to allow any pulsation in the aneurism during the night. At the hour of visit this morning (between nine and ten o'clock), on unscrewing the instrument, the pulsation of the aneurism was found to have ceased, and the tumour was hard, solid, and circumscribed. An enlarged collateral vessel, running down the centre of the popliteal space, was felt faintly beating low down in this part; the enlarged articular arteries about the knee were not felt, and the patient had experienced no pain about the joint or in the leg. He was directed to remain in bed, and to continue moderate pressure.

10th.-The compressing instruments have not been applied for some days ; the patient is quite free from pain; and the swelling of the limb perceptible on his admission has diminished. He has been kept in bed, and the diet has been gradually improved, so that he is now nearly upon full diet. The aneurismal tumour is solid and firm, the integuments covering it are loose now, and the ham-string tendons are not stretched as they were. Neither the anterior tibial artery upon the dorsum of the foot, nor the posterior tibial can be felt, nor is the pulsation of the articular arteries about the knee perceptible; the only vessel felt being a branch which runs over the centre of the popliteal space.

17th.-The patient has remained in bed since the date of the last report; he does not suffer the slightest inconvenience, and the tumour has diminished a little in size.

22d.-The patient was permitted to get up to-day; the limb, he says, feels somewhat stiff, and the ankle is slightly œdematous; in other respects he is perfectly well.

January 2d.-The patient feels no inconvenience now of any kind; the aneurismal tumour is hard, solid, and smaller. In order to promote absorption, he was directed to rub the ham with the ointment of hydriodate of potass, to which a little mercurial ointment was added.

28th.-The patient returned to the country to-day; the limb is as strong as the other; and his general health is very good. 
This makes the thirty-sixth case of external aneurism, treated by compression, in Dublin, between the years 1843 and 1850 inclusive, as will appear from the following table, containing a list of these cases; in which, under separate heads, I have given the situation of the aneurism, the hospital or other locality where the patient was treated, the name of the surgeon who had the management of the case, the result, and the name of the journal in which the case was published, with some observations in reference to the subsequent history of these patients. 


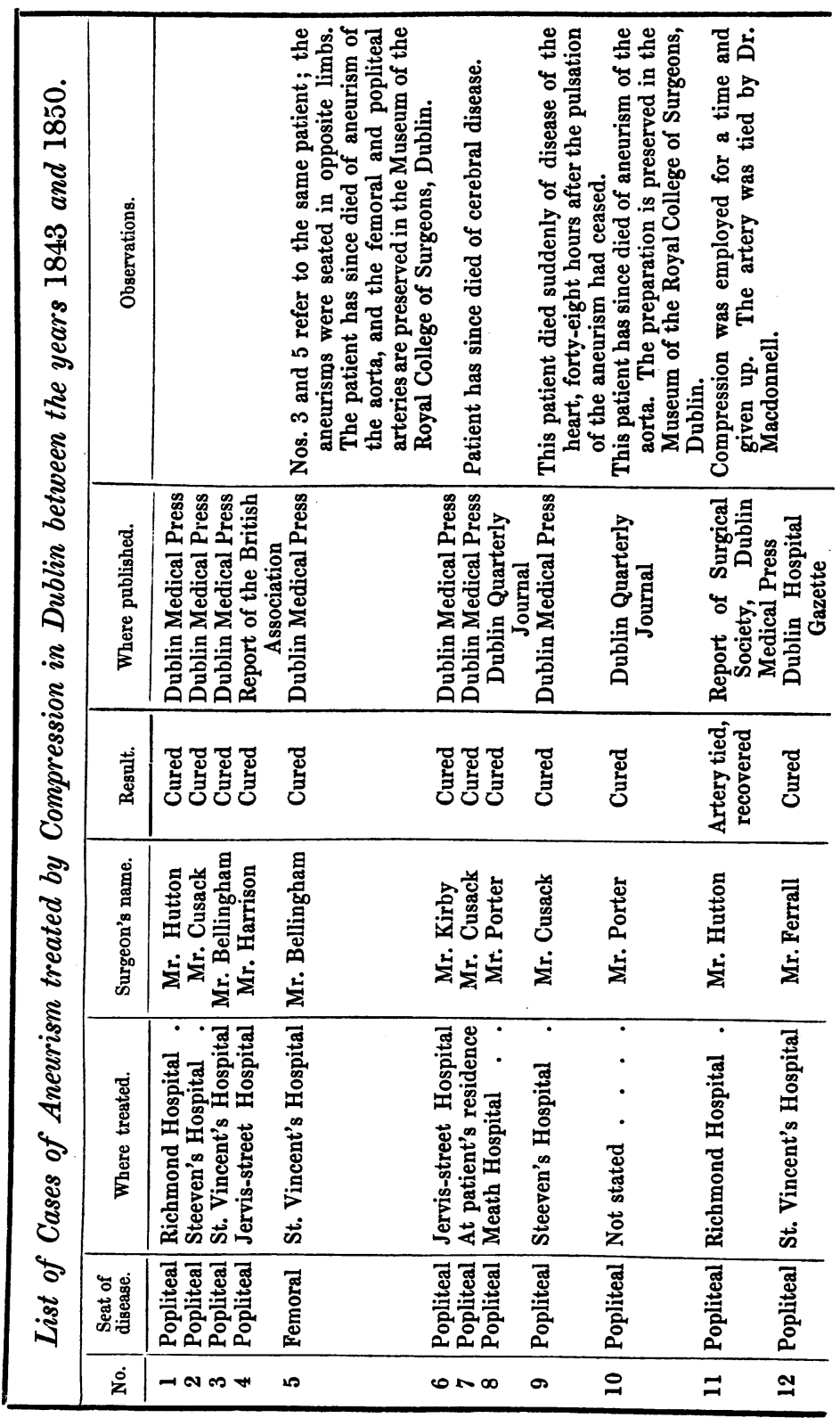




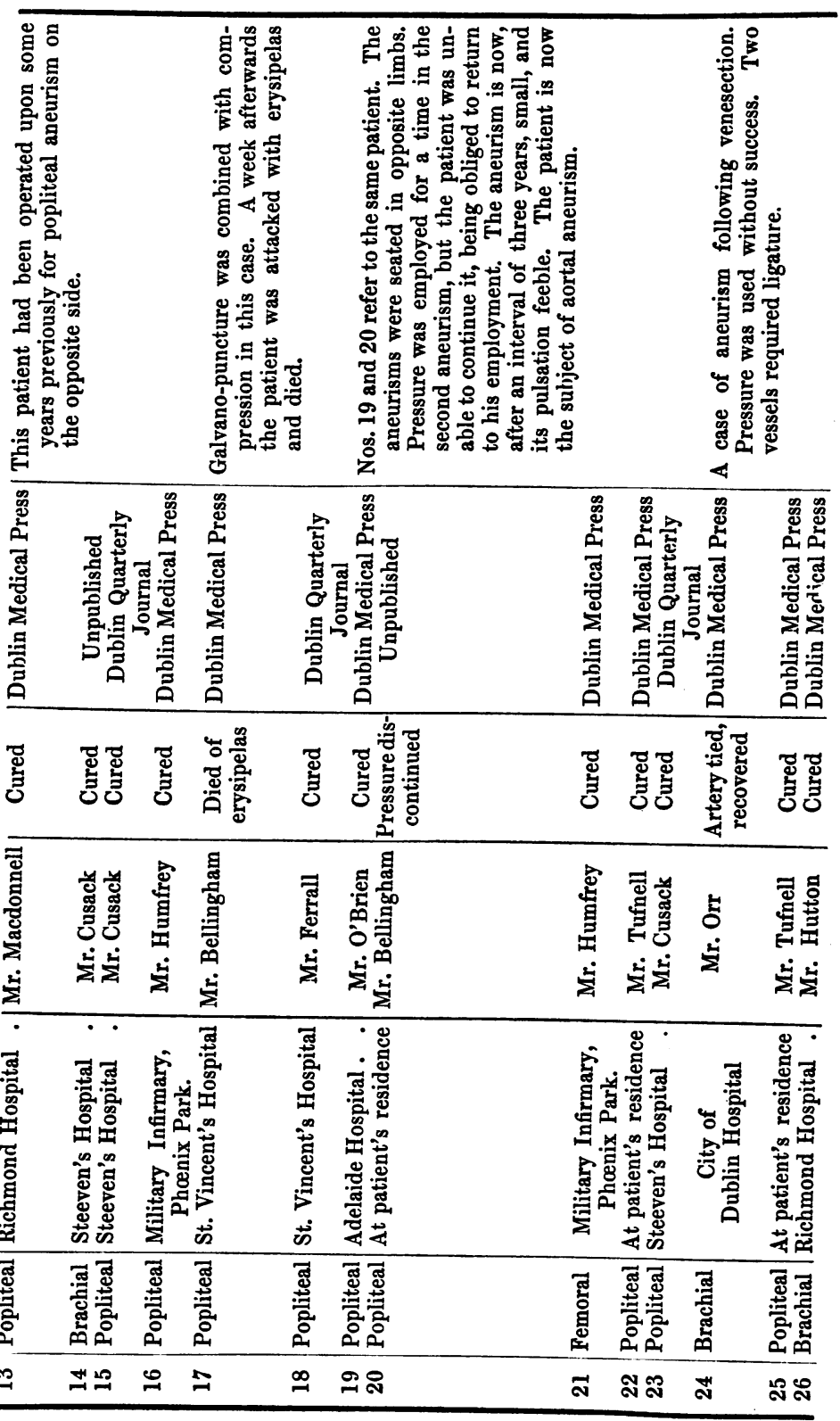




\begin{tabular}{|c|c|c|c|c|c|c|c|}
\hline 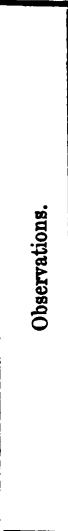 & 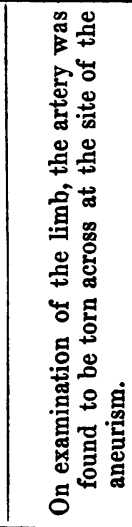 & \multicolumn{6}{|c|}{ 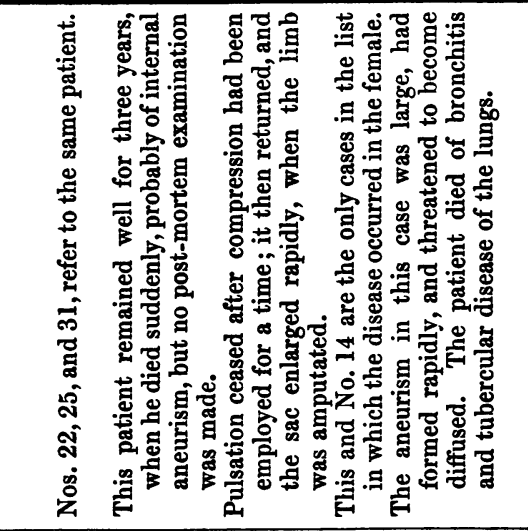 } \\
\hline 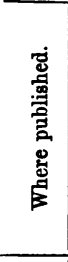 & 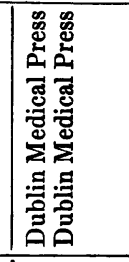 & 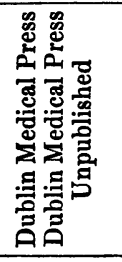 & 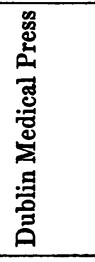 & 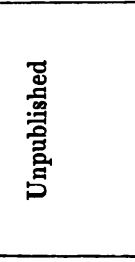 & 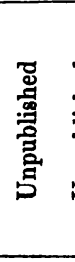 & & 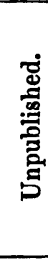 \\
\hline 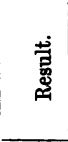 & 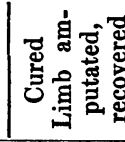 & 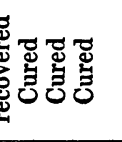 & ప్ّ & 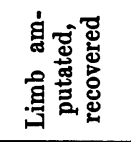 & & 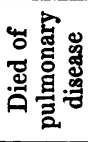 & むัّ \\
\hline 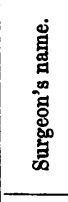 & 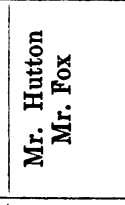 & 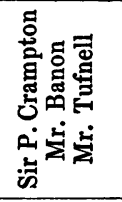 & 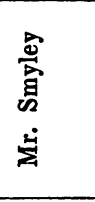 & 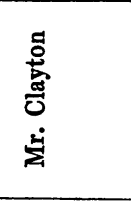 & & 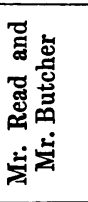 & 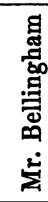 \\
\hline 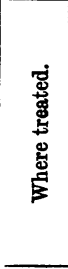 & 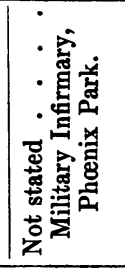 & 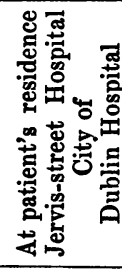 & 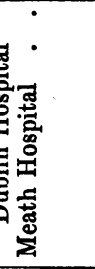 & 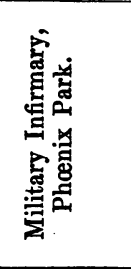 & 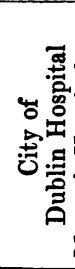 & & 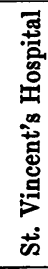 \\
\hline 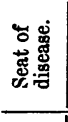 & 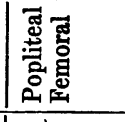 & 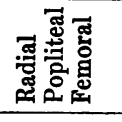 & 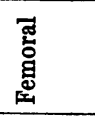 & 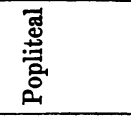 & 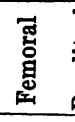 & & 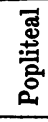 \\
\hline$\dot{\Delta}$ & ลิ & సిల్లై & శ్ & 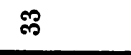 & & & is \\
\hline
\end{tabular}


The foregoing table contains a list of all the cases of popliteal or femoral aneurism, treated by compression, in Dublin, during the last seven years. It includes, also, I believe, every case of aneurism in these vessels, admitted into the Dublin Hospitals, or treated in Dublin within that period, with the exception of four ; viz., a case of femoral aneurism where compression was not employed at all, and the artery was tied with success; a case of large popliteal aneurism, where the sac had formed a communication with the knee-joint, and the limb was amputated; a case of traumatic aneurism high up in the femoral artery, where the vessel was tied with success ; and a case of varicose aneurism in the thigh, to which neither compression nor the ligature was adopted.

The table has been limited to the cases treated in Dublin, partly because the practice may be said to have originated there, and, without intending anything disparaging to other places, or other surgeons, is perhaps best understood there; and the most improved instruments for making compression have been employed there. It appeared to me, likewise, that the value of this method of treating aneurism would be best illustrated by a table of the Dublin cases, because these were not selected cases, but almost every patient labouring under popliteal or femoral aneurism, admitted into the hospitals in Dublin, since the year 1843, has been treated by compression. Besides, it was from Dublin alone that I could give a list of all the cases which came under treatment during the period in question; or, that I could vouch for the accuracy of the details, or for the permanence of the cures, which I am enabled to do, as I had the opportunity of seeing the majority of the cases given in the table, while under treatment; of assisting in the treatment of some of them ; and of examining the diseased parts, either when the patient died subsequently, or when amputation of the limb was had recourse to.

This list, it will be observed, includes 32 cases of popliteal and femoral aneurism, viz., 6 of femoral and 26 of popliteal aneurism, 3 cases of brachial, and 1 of radial aneurism. 
Of the 3 cases of brachial aneurism, 2 were cured by compression ; in the third a high bifurcation of the brachial artery existed, and when the operation came to be performed two vessels required to be tied : all three were examples of traumatic aneurism. The radial aneurism was also an example of traumatic aneurism, and was cured by compression. Of the 6 cases of femoral aneurism, 5 were cured by compression; the sixth was a case of diffuse aneurism, in which the ligature would equally have failed, and in which amputation of the limb was the only resource.

Of the 26 cases of popliteal aneurism, 21 were perfectly cured; one of them, however, (No. 9,) died, as I have observed, suddeuly, owing to organic disease of the heart, forty-eight hours after the cessation of pulsation in the aneurism. Of the remaining five cases, one (No. 20) was unable to continue the compression, being obliged to return to his employment; and now, after an interval of three years, during which the patient has been constantly occupied in a laborious employment, he has never suffered any inconvenience from it; the tumour is smaller, and its pulsation more feeble. In another (No. 11), compression was discontinued, and the artery tied, the patient recovering. In a third (No. 17) galvano-puncture was combined with compression; the patient soon afterwards was attacked with erysipelas in a very severe form and died. In a fourth (No. 33) the aneurism enlarged considerably, pressure having been discontinued, and the limb was amputated. And in the fifth (No. 35) the patient died of pulmonary disease, and the aneurism, on examination after death, was found to be in a great measure filled up by fibrin. I may add, that the only two cases in the list in which amputation was performed, occurred in a military, not a civil hospital, where the treatment was of course conducted by the military medical officer, temporarily in charge.

In three of the cases in this list, (Nos. 3 and 5, 19 and 20, and 22,25 , and 31 ,) the patients underwent the treatment by compression for aneurism in opposite limbs, one of them was treated three times, viz., twice for popliteal aneurism in 
opposite limbs, and once for femoral, each time with success. One of the patients (No. 13) had undergone the operation by ligature for popliteal aneurism in the opposite limb, some years previously. Four of the patients in this list (Nos. 3 and $5,7,10$, and 32 ) have since died, two of aortal aneurism, one probably also of internal aneurism, and one of cerebral disease; another ${ }^{1}$ (No. 19 and 20) is at present labouring under aneurism of the aorta. Only two of the patients in the foregoing list (Nos. 14, and 34) were females.

When compression first came to be employed in Dublin as a mode of treating aneurism, it was objected that the disease would be liable to return : in no case, however, marked cured in the forgoing list has any thing of the kind occurred; the cure in all has been permanent, and the patients have gained the perfect use of the limb. Indeed, from the manner in which compression brings about the cure of aneurism, it is clear that the disease cannot return in the same part of the vessel, and, in the opportunities which I have had of examining the parts some time subsequently, owing to the patient's death from other causes, the artery has invariably been found to be obliterated at the seat of the aneurism, and converted into an impervious, ligamentous band at the part; while the portion of the artery upon which pressure had been made, preserved its normal condition, and neither it nor the vein (which, from its proximity to, and close connection with the artery, must always, in cases of femoral or popliteal aneurism, be compressed with the artery,) presented, in a single instance, any deviation from the normal and healthy state.

When we contrast the results of the treatment of aneurism by compression, with the results of the ligature in aneurism of the same arteries, as furnished by the most recent statistical tables, the comparison appears very favorable to compression: thus, the table which $I$ have given contains 36 cases; in 29

1 This patient has since died, and I had the opportunity of making an examination of the limbs. 
a cure was effected by compression; of the remaining 7 cases the artery was tied in 2 , the patients recovering. In 1 , pressure was discontinued, the aneurism subsequently diminished in size, and the patient had the perfect use of the limb for three years, when symptoms of aneurism of the aorta supervened, and compelled him to give up his employ. ment. In 2 , the limb was amputated, the patients recovering; and in the remaining two, death occurred, in one from pulmonary disease, in the other from a severe attack of erysipelas; but in both, the local disease was very nearly cured, the aneurismal sacs being almost completely filled by fibrine deposited in concentric layers.

If we now turn to the statistics of the ligature, furnished by the most recent statistical tables, the results appear much less satisfactory. Thus, in a table given by Dr. Norris in the 'American Journal of Medical Science,' 188 cases where the femoral artery was tied for aneurism are reported, (in 155 for popliteal aneurism, and the following are the results :-

No. of cases.

Cured.

Died.

188

142

46

The majority of the deaths was owing to gangrene, secondary hæmorrhage, phlebitis, tetanus, \&c.; while six of the patients who recovered underwent amputation of the limb.

Another table is contained in Mr. Crisp's 'Treatise on the Diseases of the Blood-vessels.' This includes 119 cases of popliteal aneurism treated by the ligature, with the following results :-

No. of cases.

Cured.

Died.

119

103

16

The deaths were the result of secondary hæmorrhage, gangrene, phlebitis, tetanus, delirium tremens, the shock of the operation, and suppuration in the sac. Six of the patients under the head "cured," underwent amputation of 
the limb after the ligature; and six others recovered, notwithstanding the occurrence of gangrene, secondary hæmorrhage, or suppuration in the sac.

The summary of the results of the ligature, in the cases of popliteal and femoral aneurism, contained in these tables, would appear to be even too favorable to the ligature. The same cases are included in both; and it is evident that they can form only a moiety of the cases of aneurism of these vessels, in which the ligature has been used, during the period in question, (fully half a century.) When we consider, in addition, with what unwillingness cases which turn out unfavorably are published, it seems highly probable, that, if we were in possession of full statistics of all the operations for aneurism performed upon these vessels, the per centage of deaths would be found to be much greater. Even taking the statistics of the ligature as we find them, if we contrast its results with that of compression in the same forms of aneurism, we perceive a remarkable immunity from risk, in the one method of treatment, and a considerable amount of risk attending the operation in the other. Thus, the great majority of the deaths after the ligature were owing to secondary hæmorrhage, gangrene, phlebitis, tetanus, and delirium tremens, in other words, directly to the result of the operation; while in the treatment by compression, all those accidents were avoided, not one of them having occurred in the thirty-six cases contained in my table. The two deaths in it were, I may say, from causes independent of the treatment; the one having been from erysipelas, which was prevalent in the hospital at the time; the other from pulmonary disease. Both were likewise men of broken-down constitutions, with diseased heart and arteries; very unfavorable subjects, consequently, for operation, and in whom, if the ligature had been used, it would most probably have proved a failure.

Although compression has proved eminently successful in Dublin, as a mode of treating certain forms of aneurism, and has so completely superseded the ligature there, that 
the latter has not been had recourse to for several years past in a single case of popliteal aneurism, either in hospital or in private practice, it must be confessed that the reports from most other places are less favorable; it is therefore scarcely unreasonable to conclude that compression cannot have been employed in the same way, that equally effective instruments cannot have been used, or that the same precautions cannot have been taken to ensure success. Indeed, a perusal of the cases which have been published of the failure of compression in the hands of surgeons out of Dublin, (which have been put so prominently forward in some places) has satisfied me, that the failure in the majority resulted either from the imperfection of the instruments employed, from an unnecessary degree of pressure having been used, or from its having been made in an imperfect manner, from tight bandages having been applied to the limb while pressure was maintained upon the main artery, from the treatment having been commenced without due attention to constitutional measures, or finally from compression having been used in cases where the ligature would equally have failed.

It is unnecessary to say that compression is not employed at the present day with the object of endeavouring to obliterate the artery at the point compressed, if it were, few patients would have been found possessed of the fortitude to submit to the pain such an amount of pressure would occasion. I do not, however, mean to assert, that compression is a painless process; but $I$ do say that the pain may be much increased, and that the patient may be, and often has been, put to a great deal of unnecessary pain, and may refuse to continue its use from this cause alone.

I do not wish it, either, to be supposed that compression is advocated in every form of aneurism, or that it is supposed to be capable of effecting a cure in every variety of the disease, as, for instance, in diffused aneurism, or where the aneurismal sac had been allowed to attain an extremely large size, or where the sac of a popliteal aneurism had formed a communication with the knee joint, or had caused 
erosion of the bone; in fact in cases where amputation is perhaps our only resource.

Compression, as a mode of treatment in aneurism, is advocated mainly as a substitute for the ligature, and for cases to which the ligature is applicable, provided the sac is so situated that compression can be made upon the artery at its cardiac side, as well as for cases where the arterial tunics are diseased, and the ligature would be consequently very likely to fail. It is advocated because pathology has shown that it brings about the cure of aneurism by the very mode in which nature, under the most favorable circumstances, effects this object, and because experience has proved it to be a safe and certain method of treatment, while all experience has shown the ligature to be directly the reverse.

If we expect, however, to be successful, and if we hope to effect a cure speedily, our efforts must be mainly directed to the means whereby pressure may be maintained with as little inconvenience as possible to the patient, which will depend in a great measure upon the kind of apparatus used, and its completeness; upon the manner in which the pressure is maintained ; and, upon the constitutional measures adopted previous to the treatment being commenced, and while it is in progress.

A good deal will also depend upon the intelligence and tractability of the patient. In a mode of treatment which usually occupies several days, and sometimes more, it is not easy to have a constant surveillance; and if the patient unscrews the instrument the moment the surgeon's back is turned, the cure must of course be retarded; or, if he is too stupid to understand the manner of using the compressing force, or the object with which it is applied, the pressure will be imperfectly maintained, and at each visit the surgeon will probably find the pad of the instrument resting upon any other part than the artery.

With respect to the instruments, I need only observe that those which are available now, are in many respects superior to those we were in possession of a few years ago, the un- 
yielding force of the screw has been superseded by the elastic force of the vulcanized India-rubber bnads, an improvement for which we are indebted to Dr. Carte; the treatment, consequently, has been much simplified, and the pressure can be borne with less inconvenience by the patient. While upon this point, I may observe that it is scarcely doing justice to this method of treating aneurism, to undertake it without being provided with a proper apparatus for carrying it out; yet this would appear not unfrequently to have occurred in the trials of compression made in other places, and these very cases have been afterwards published as examples of the failure of compression, and have been made a ground for decrying this method of treatment, while the fault lay, in a great measure, with the surgeon who commenced the treatment without having proper instruments at hand for carrying it out.

In the list which I have given, of the cases of aneurism treated by compression in Dublin, there were few in which the treatment was so quickly successful, and in which the pulsation of the aneurism ceased within so short a period, as in that just detailed. This fortunate result may be attributed, in part, to the constitutional treatment to which the patient was subjected, previous to the application of the compressing instruments, and as this was the first case in which constitutional measures of the kind were combined with the local measures, and as it promises to be an important agent in the treatment, in future, I may be permitted to make a few remarks upon it.

When the patient was admitted into the hospital, his pulse, as I have said, was strong and incompressible. In such a state of the circulation, a much greater amount of pressure would have been found necessary in order to stop the pulsation of the aneurism than was required in his case; greater pain would unavoidably have been inflicted on him, and greater difficulty would have been experienced in maintaining the pressure from this very cause.

With the view of diminishing the hardness and incom- 
pressibility of the pulse, bleeding has been sometimes advantageously premised, particularly when the patient was young and plethoric. I was unwilling, however, to bleed this patient, as although muscular and stout, he did not appear to be a favorable subject for the abstraction of blood. I preferred, therefore, adopting a plan of treatment, which I have employed with advantage in some cases of aneurism of the aorta, under the use of which $I$ have found the pulse to become soft, small, and compressible, and its frequency to be diminished. This consists in an extremely restricted diet, especially as respects fluids, the utmost amount allowed being but six ounces in the twenty-four hours, with eight ounces of solid food in the same period, combined with the daily or frequent use of some hydragogue cathartic, the patient being at the same time strictly confined to the horizontal posture.

Under the use of this regimen, continued for some days, the hardness and incompressibility of this patient's pulse diminished and it became slower; the compressing instruments were consequently borne with little inconvenience. At the same time, the watery constituents of the blood being diminished, this fluid became more dense, by which the deposition of its fibrin, in its passage through the aneurismal sac, was favoured; this began early to be deposited, and before twenty-four hours had so much increased that the pulsation of the aneurism ceased. Had compression, on the other hand, been at once commenced without any preparatory treatment, much stronger pressure would have been required; and as the pain is, in some measure, in the ratio to the degree of pressure, this patient would have been subjected to much unnecessary pain while the treatment would unavoidably have been more prolonged.

Although in many of the cases in the list which I have given, compression proved effectual without almost any preparatory treatment, and in none was any, like that adopted here, employed, I am of opinion that it ought to constitute a prominent item in the treatment, tending, as it most certainly does, to diminish the pain which the compressing 
instruments occasion, as well as to shorten the period required for their employment. And I am convinced that if in future, constitutional measures analogous to those employed in this case, are made to precede the local treatment, and that the latter is fully and fairly carried out, no case to which compression is applicable will be found to resist, and those who are most sceptical will be obliged to acknowledge its superiority over the ligature. 